clavian veins, superior vena cava, and intracerebral venous sinuses showed no evidence of thrombosis or anatomical abnormality. Cystic changes were evident in both occipital poles of the brain.

\section{Discussion}

Percutaneous central venous catheters provide reliable venous access in the newborn ${ }^{1}$ and are widely used for delivering parenteral nutrition. ${ }^{2}$ In contrast to the major complication of sepsis, serious mechanical catheter related problems are rarely encountered. ${ }^{34}$ Two previous cases of parenteral feeding solution accumulating in the subdural space have been described in preterm infants but neither case came to postmortem examination. ${ }^{56}$ In one of these patients the catheter tip was situated in the superior vena cava and swelling of the neck several hours after onset of neurological symptoms suggested a relationship between subdural effusion and superior vena cava thrombosis. ${ }^{5}$ In the second case report the catheter tip was left in the temporal vein with no clinical evidence of vessel occlusion, although the authors postulated a probable septic thrombosis. ${ }^{6}$

In the only case that has been subjected to a full postmortem examination our findings indicate that a subdural collection of intravenous feeding fluid may occur in the absence of venous thrombosis or apparent anatomical abnormality. This being the case, we would agree with suggestions that retrograde flow of parenteral nutrition infusion along the internal jugular vein to the transverse sinus, sagittal sinus and, via a ruptured bridging vein into the subdural space, is the likely mechanism. Together with bronchopulmonary dysplasia, raised pulmonary vascular resistance might have been the cause of increased venous pressure giving rise to retrograde flow.

Subdural accumulation of intravenous feeding fluid appears to be a very rare complication of parenteral nutrition given via a misplaced central venous catheter. It would seem prudent whenever possible to site the tip of the catheter within the mid right atrium in an attempt to minimise the risk of venous thrombosis or retrograde flow. Acute onset of neurological symptoms in an infant with a central venous catheter should raise the possibility of a subdural effusion.

We would like to thank Dr S Variand for his help with the postmortem examination of this case.

1 Shaw JCL. Parenteral nutrition in the management of sick, low birthweight infants. Pediatr Clin North Am 1973;20: 333-58.

2 Puntis JWL. Percutaneous insertion of Silastic central venous feeding catheters. Intensive Therapy and Clinical Monitoring 1987;8:7-10.

3 Gladman G, Sinha S, Sims DG, Chiswick ML. Staphylococcus epidermidis and retention of neonatal percutaneous
central venous catheters. Arch Dis Child 1990;65:234-5. 4 Rubin S, Hewson P, Roberton NRC. Pulmonary complications of total parenteral nutrition in a neonate. $\mathcal{F} R$ Soc Med

5 Stine MJ, Harris $H$. Subdural collection of intravenous fat emulsion in a neonate. Clin Pediatr (Phila) 1985;24:40-1. 6 Young S, MacMahon P, Kovar IZ. Subdural intravenous fat collection: an unusual complication of central intravenous feeding in the neonate. Fournal of Parenteral and Enteral Nutrition 1989;13:661-2.

\title{
A national survey of nebuliser use
}

\author{
Helena J Childs, Carol A Dezateux
} Epidemiology, Institute of Child Health, 30 Guilford Street, London WCIN 1EH Helena J Childs Carol A Dezateux Correspondence to: Ms Childs.

Accepted 2 August 1991
Department of Paediatric

\begin{abstract}
Nebuliser drug delivery units were reused in $15 \%$ of paediatric wards participating in a national survey, while routine servicing and written information was provided by only half the wards issuing home nebulisers. Written information should be developed as a national resource, and further research on optimal cleaning practices is required.
\end{abstract}

Nebulisers are commonly used to treat children with severe asthma or cystic fibrosis in hospital and are increasingly prescribed for home treatment of very young children and those with chronic disease. While this may reduce the need for hospital admission, particularly of children with chronic conditions, it has been suggested (Arch Dis Child 1991;66:1351-3) that over-reliance on nebulised bronchodilators and inadequate supervision and education may increase the risk of life threatening asthmatic episodes. ${ }^{1}$ Nebuliser drug delivery units are manufactured for use by a single patient only, and though reuse may save money, potential hazards include bacterial contamination ${ }^{2}$ and loss of efficiency of drug delivery. A recent King's Fund conference concluded that reuse of items manufactured for single patient use was undesirable and recommended development of guidelines governing reuse at district levels. ${ }^{3}$

This survey aimed to determine current cleaning and reuse practices and to obtain information on the advice and support given to parents issued with a home nebuliser in a nationally representative sample of paediatric wards in England. We also sought to establish whether guidelines governing reuse were available at district health authority level. 
Methods

Three district general hospitals selected at random from each of the 14 regional health authorities in England, and all regional, teaching, and specialist hospitals were included. Verbal informed consent to take part was obtained from the nurse in charge of each ward and a standard questionnaire administered at a subsequent prearranged time. All contact was by telephone (HJC).

Information was obtained for each ward on the following: (i) number of beds, (ii) number of children with asthma and cystic fibrosis admitted in the preceding calendar month; (iii) type of nebuliser drug delivery unit used, (iv) cleaning and reuse practices during an individual child's course of treatment and between children, and (v) and existence of a written ward policy for cleaning and reuse of drug delivery units. Staff responsible for issuing home nebulisers were identified, and details of advice on medical treatment, written material available for parents and children, as well as cleaning, operating, and servicing of home nebulisers obtained.

All district general managers in England were contacted by post to establish whether written guidelines on the cleaning and reuse of drug delivery units were available in their district.

\section{Results}

Two of the 77 wards contacted between February and August 1990 had closed and the nurse in charge of one ward refused to participate. Questionnaires were completed for 74 wards (96\%) of which 43 were general paediatric, 29 medical paediatric, and two specialist respiratory wards. Most wards $(n=60)$ had less than 30 beds. Information on admissions was available for 54 wards (73\%): all had admitted children with asthma and 32 had also admitted children with cystic fibrosis in the calendar month preceding the questionnaire.

The System 22 or Hudson nebuliser drug delivery units were most frequently used (44 wards). The drug delivery unit was not changed in 56 wards $(76 \%)$ during an individual child's course of treatment. Between drug doses, the drug delivery unit was not cleaned in 29 wards $(39 \%)$, while in the remaining 45 the most frequent method of cleaning was washing with soap and water and drying. The drug delivery unit was kept at the child's bedside in 69 wards, and in 54 of these it was stored open to the atmosphere.

Although discarded in most wards when an individual child's course of treatment had been completed, the drug delivery unit was reused for another child in 11 wards (15\%), 10 of which were admitting children with cystic fibrosis as well as asthma. Before reuse, the drug delivery unit was cleaned with soap and water and dried (six wards), or cleaned in sterilising solution (two wards), or sent to be resterilised (three wards). A ward policy on cleaning and reuse of nebuliser drug delivery units was available in only 17 wards.

All but two wards issued home nebulisers. In $52(72 \%)$ of these, nursing and medical staff were jointly involved in educating parents on issues such as drug dose, frequency of doses and when to seek medical advice. In 51 wards (71\%), nursing staff alone were responsible for instructing parents on the operation of the home nebuliser and cleaning of the drug delivery unit. Written information for parents was provided in only 42 wards (58\%), and for children in only three wards. Nebulisers were not recalled for servicing in 33 wards (46\%) and only $23(32 \%)$ met the British Thoracic Society recommendations of servicing twice a year. ${ }^{4}$

The regional, teaching, and specialist hospitals did not differ from the district general hospitals in any of the above practices.

Written replies were received from 177 of the 192 general managers contacted (92\%): 110 district health authorities (62\%) had no policy on cleaning and reuse of nebuliser drug delivery units. Information from 51 wards could be linked to that obtained from the relevant district health authority. Although general managers reported that a district policy was available for use in 22 of these wards, staff in only seven wards were aware of the existence of such a policy.

\section{Discussion}

Given the reported increase in hospital admissions for asthma in the UK and the rising number of nebulisers bought or supplied for use at home,${ }^{5}$ the findings from this survey give cause for concern. Although manufactured specifically for single patient use, drug delivery units were reused in a number of paediatric wards. Almost all of these admitted children with cystic fibrosis, and reuse may increase the risk of bacterial cross infection to all children receiving nebulised therapy. ${ }^{2}{ }^{6}$ It is unclear to what extent a drug delivery unit may be reused, even for a single child, and to what extent its reuse may impair the efficiency of drug delivery. This could be of particular importance in the home when repeated use may occur for many months. Further research is needed on the optimal duration of use of the drug delivery unit.

It was of concern that there was no routine servicing of home nebulisers in almost half the wards, and servicing twice a year in only one third. District general managers need to be alerted to recently published guidelines on nebuliser maintenance. ${ }^{4}$ No policy on cleaning and reuse was available in more than half of district health authorities, and, even when available, nurses in charge of paediatric wards were frequently unaware of its existence.

There is a dearth of written information for parents and children issued with home nebulisers. Producing clear written information is a major undertaking and this should be developed as a national resource for parents as well as children, who, as they grow up, become increasingly responsible for their own treatment. We would like to thank all of the nurses and district general
managers who participated in this survey and Mark Whiting for managers who participated in this survey and Mark Whiting for commenting on questionnaire design. HJC was supported by the National Asthma Campaign and CAD by a Wellcome Research Training Fellowship in Clinical Epidemiology and the National Asthma Campaign. 
1 Laroche CM, Harries AVK, Newton RCF, et al. Domiciliary nebulisers in asthma: a district survey. $B M \mathcal{F} 1985 ; 290$ : 1611-3.

2 Barnes KL, Clifford R, Holgate ST, et al. Bacterial contamination of home nebulisers. BMf̈ 1987;295:812.

3 King's Fund College. Reuse of sterile, single use and disposable equipment in the NHS. London: King Edward's Hospital

4 British Thoracic Society, Research Unit of the Royal College of Physicians of London, King's Fund Centre, National Asthma Campaign. Guidelines for management of asthma in adults. BMF 1990;301:651-3.

5 Bradley N, Watkins S. Survey of equipment in general practice. BMF 1989;299:435-6.

6 Wexler MR, Rhame FS, Blumenthal MN, Cameron SB, June BA, Fish LA. Transmission of Gram-negative bacilli to asthmatic children via home nebulisers. Ann Allergy 1991;66:267-71.

\title{
Familial infantile oesophageal achalasia
}

\author{
T K Kaar, R Waldron, M S Ashraf, J B G Watson, M O’Neill, W O Kirwan
}

Republic of Ireland

T K Kaar

$\mathrm{R}$ Waldron

M S Ashraf

J B G Watson

M O'Neill

W O Kirwan

Correspondence to:

Dr Watson.

Accepted 16 July 1991

(Arch Dis Child 1991;66:1353-4)

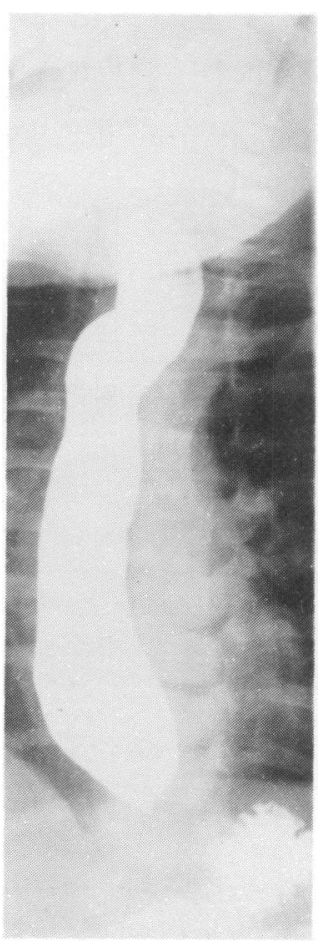

Barium swallow in case 2 showing achalasia.
Oesophageal achalasia is a disease of unknown aetiology characterised by a functional obstruction of the lower oesophagus due to failure of relaxation of the lower oesophageal sphincter and altered motility of the body and distal oesophagus. It is an uncommon disease, usually presenting in adult life, and its occurrence in childhood is rare. Occasionally it occurs in a familial form. We report two male siblings who presented with oesophageal achalasia during infancy and who underwent corrective surgical treatment.

\section{Case reports}

The parents of the children were first cousins. Clinical examination and laboratory investigations excluded any syndromic type of achalasia in both cases.

\section{CASE 1}

A first born boy presented at 5 months of age with recurrent respiratory tract infection, abdominal distension, and failure to thrive. A barium swallow performed showed typical features of achalasia. At operation at the age of 10 months the child underwent a transabdominal modified Heller's anterior oesophagomyotomy of $8 \mathrm{~cm}$ length and Nissen fundoplication. The child is now well and thriving after three years of follow up.

\section{CASE 2}

The second and only other child in the family was also male and presented at 8 months of age with a history of respiratory tract infections,

\begin{abstract}
Department of Paediatrics, Regional Hospital, Wilton,

Cork,

Abstract

Oesophageal achalasia is uncommon in children and in its familial form it is a rarity. The presentation and management of two male siblings who presented with oesophageal achalasia as infants are reported. A high degree of consanguinity in the parents of the children existed, suggesting autosomal recessive transmission.
\end{abstract}

regurgitation and vomiting, and failure to thrive since birth. Barium swallow was performed which again showed the features of well established achalasia (figure). Operation was performed at 10 months of age and again a transabdominal modified Heller's anterior oesophagomyotomy $(8.5 \mathrm{~cm}$ long) with Nissen fundoplication was performed. The child made a good recovery postoperatively and is now thriving after six months of follow up.

\section{Discussion}

Achalasia of the oesophagus is uncommon with an incidence estimated at approximately one per 100000 population per year. ${ }^{1}$ In children achalasia is rare with only $2 \%$ of all cases presenting before the age of 6 years. ${ }^{2}$

A familial form of achalasia presenting in infant siblings was first described by Thibert et al in $1965 .{ }^{3}$ Before this in 1962 Tyce and Brough reported a family with multiple diseases inherited including mental retardation, oesophageal achalasia, speech disorder, and neurological diseases. ${ }^{4}$ Dayalan et al in 1971 reported the presentation of three siblings with achalasia during the first year of life. ${ }^{5}$ In the case they reported the parents of the affected children were closely consanguineous, the father being the maternal uncle of the mother; there were two boys and one girl.

Westley et al in 1975 proposed that infantile achalasia is inherited as an autosomal recessive disorder when describing its occurrence in an Apache Indian kindred. ${ }^{6}$ He postulated that the high degree of inbreeding allowed a rare recessive gene to be expressed in several members. The existence of consanguinity in the parents of the children in this report lends further weight to this argument. Although vertical transmission of oesophageal achalasia has been described, the lack of consistent vertical transmission is also thought to indicate a probable autosomal recessive gene disorder. Further support for autosomal recessive inheritance is the well documented occurrence of achalasia in association with other conditions having a similar mode of inheritance. 